\title{
A Root-MUSIC-Like Direction Finding Method for Cyclostationary Signals
}

\author{
Pascal Chargé \\ LESIA, DGEI, INSA Toulouse, 135 Avenue de Rangueil, 31077 Toulouse Cedex 4, France \\ Email: pascal.charge@insa-toulouse.fr \\ Yide Wang \\ IREENA/SETRA, École Polytechnique de l'Université de Nantes, La Chantrerie, 44306 Nantes, France \\ Email: yide.wang@polytech.univ-nantes.fr
}

Received 4 December 2003; Revised 2 June 2004

\begin{abstract}
We propose a new root-MUSIC-like direction finding algorithm that exploits cyclostationarity in order to improve the directionof-arrival estimation. The proposed cyclic method is signal selective, it allows to increase the resolution power and the noise robustness significantly, and it is also able to handle more sources than the number of sensors. Computer simulations are used to show the performance of the algorithm.
\end{abstract}

Keywords and phrases: array processing, direction finding, MUSIC, cyclostationary signals.

\section{INTRODUCTION}

The aim of this paper is the estimation of the direction-ofarrival (DOA) of impinging signals in the telecommunications systems area, where almost all signals exhibit the cyclostationarity property [1]. The cyclostationarity has been first introduced into array processing by Gardner [2]. We can find in the literature several algorithms (see [1]) that exploit cyclostationarity to improve the performances of the conventional methods. Instead of using the correlation matrix as in the conventional methods, these algorithms require the estimation of the cyclic correlation matrix that reflects the cyclostationarity of incoming signals, assuming they have baud rates and/or are carrier modulated signals as they would be in radar and radio communication applications. Recently, an extended cyclic MUSIC algorithm has been proposed in [3] that provides a rather good estimation performance. In [4] an extended root-MUSIC (extension of the rootMUSIC [5]) algorithm has been proposed in the noncircular source case.

In this paper, we propose a new extended cyclic direction finding method that allows to select desired signals and to ignore interferences, by exploiting the cyclostationarity property of the signals of interest (SOI). The proposed method is inspired from the extended root-MUSIC method [4], and then is restricted to linear uniformly spaced arrays. But it has the distinct advantage over [3] in that it does not require a search over parameter space. Instead, our algorithm here requires calculation of the roots of a polynomial, which is a simple process and has low computation cost.

\section{DATA MODEL}

In this paper, we consider a uniform linear array of $L$ antennas. Suppose there are $K$ electromagnetic waves impinging on the array from angular directions $\theta_{k}, k=1, \ldots, K$. The incident waves are assumed to be plane waves, as generated from far-field point sources. Furthermore, the signals are assumed to be narrowband. In our study, we assume that $K_{\alpha}$ sources emit cyclostationary signals with cycle frequency $\alpha$ (with $K_{\alpha} \leq K$ ). In the following, we consider that $\mathbf{s}(t)$ contains only the $K_{\alpha}$ signals that exhibit cycle frequency $\alpha$, and all of the remaining $K-K_{\alpha}$ signals (that have no cycle frequency $\alpha)$ and any noise are lumped into a vector $\mathbf{i}(t)$. Using this assumption, the signal received by the array from the emitting narrowband sources can be written as

$$
\mathbf{z}(t)=\mathbf{A s}(t)+\mathbf{i}(t),
$$

where the vector $\mathbf{s}(t)=\left[s_{1}(t), \ldots, s_{K_{\alpha}}(t)\right]^{T}$ contains the temporal signals that have cycle frequency $\alpha$, while the vector $\mathbf{i}(t)$ represents interfering sources and noise. The matrix $\mathbf{A}=\left[\mathbf{a}\left(\theta_{1}\right), \ldots, \mathbf{a}\left(\theta_{K_{\alpha}}\right)\right]$ contains the steering vectors of the impinging SOI. We assume that the received signals are sampled at $N$ distinct times $t_{n}, n=1,2, \ldots, N$. 
The cyclic autocorrelation matrix and the cyclic conjugate autocorrelation matrix at cycle frequency $\alpha$ for some lag parameter $\tau$ are then nonzero and can be estimated by

$$
\begin{aligned}
& \mathbf{R}_{\mathbf{z z}}^{\alpha}(\tau)=\frac{1}{N} \sum_{n=1}^{N} \mathbf{z}\left(t_{n}+\frac{\tau}{2}\right) \mathbf{z}^{H}\left(t_{n}-\frac{\tau}{2}\right) e^{-j 2 \pi \alpha t_{n}}, \\
& \mathbf{R}_{\mathbf{z z}}^{\alpha}(\tau)=\frac{1}{N} \sum_{n=1}^{N} \mathbf{z}\left(t_{n}+\frac{\tau}{2}\right) \mathbf{z}^{T}\left(t_{n}-\frac{\tau}{2}\right) e^{-j 2 \pi \alpha t_{n}},
\end{aligned}
$$

where the superscript $*$ denotes the complex conjugate, $H$ denotes the conjugate transpose, and $T$ denotes the transpose.

We form the following extended data vector:

$$
\mathbf{z}_{\mathrm{CE}}(t)=\left[\begin{array}{c}
\mathbf{z}(t) \\
\mathbf{z}^{*}(t)
\end{array}\right]
$$

The cyclic correlation matrix for this extended data model can be estimated as

$$
\mathbf{R}_{\mathrm{CE}}^{\alpha}(\tau)=\frac{1}{N} \sum_{n=1}^{N} \mathbf{I}_{2 L}^{\alpha}\left(t_{n}\right) \mathbf{z}_{\mathrm{CE}}\left(t_{n}+\frac{\tau}{2}\right) \mathbf{z}_{\mathrm{CE}}^{H}\left(t_{n}-\frac{\tau}{2}\right)
$$

where the time-dependent matrix $\mathbf{I}_{2 L}^{\alpha}(t)$ is defined by

$$
\mathbf{I}_{2 L}^{\alpha}(t)=\left[\begin{array}{cc}
\mathbf{I}_{L} e^{-j 2 \pi \alpha t} & \mathbf{0} \\
\mathbf{0} & \mathbf{I}_{L} e^{+j 2 \pi \alpha t}
\end{array}\right]
$$

and $\mathbf{I}_{L}$ is the $L$-dimensional identity matrix. This extended cyclic correlation matrix can be developed as

$$
\mathbf{R}_{\mathrm{CE}}^{\alpha}(\tau)=\left[\begin{array}{cc}
\mathbf{R}_{\mathbf{z z}}^{\alpha}(\tau) & \mathbf{R}_{\mathbf{z z}^{*}}^{\alpha}(\tau) \\
\mathbf{R}_{\mathbf{z z}^{*}}^{\alpha *}(\tau) & \mathbf{R}_{\mathbf{z z}}^{\alpha *}(\tau)
\end{array}\right]
$$

where the matrices $\mathbf{R}_{\mathbf{z z}}^{\alpha}(\tau)$ and $\mathbf{R}_{\mathbf{z z}}^{\alpha}(\tau)$ are estimated by (2) and (3), respectively.

By choosing the cycle frequency parameter $\alpha$ in the estimation of the extended correlation matrix to be the cycle frequency of the $K_{\alpha}$ SOI, the contribution to the cyclic correlation matrix from the other $K-K_{\alpha}$ signals (assumed not to have the same cycle frequency) and from any noise converges to zero as the integration time used in the estimate tends to infinity.

\section{DOA ESTIMATION}

By computing the SVD of $\mathbf{R}_{\mathrm{CE}}^{\alpha}(\tau)$ similarly to the cyclic MUSIC algorithm, a left signal subspace can be defined by the $K_{\alpha}^{\prime}$ left singular vectors associated with the $K_{\alpha}^{\prime}$ nonzero singular values. These singular vectors form the column vectors of the matrix $\mathbf{U}_{s}$. In the same way a left null space is spanned by the remaining $2 L-K_{\alpha}^{\prime}$ singular vectors associated with the zero singular values of $\mathbf{R}_{\mathrm{CE}}^{\alpha}(\tau)$, and these singular vectors are the column vectors of the matrix $\mathbf{U}_{n}$.
Note that in the practice, there are no zero singular values but only small singular values, and the dimension on the signal subspace can be estimated by the MDL criterion [6]. Some cyclostationary signals have a rank two in the signal subspace spanned by column vectors of the matrix $\mathbf{U}_{s}$ and others have only a rank one, so that the dimension of the signal subspace is equal to $K_{\alpha}^{\prime}$ with $K_{\alpha} \leq K_{\alpha}^{\prime} \leq 2 K_{\alpha}$ (see [3] for more details).

From this statement, we can conclude that in the worst case if all signals have a rank two, we can only detect up to $L-1$ possible DOA. But when all signals have a rank one, the number of incoming signals that can be detected is twice that in the worst case.

As in [3], an extended steering vector corresponding to this data model can be defined as follows:

$$
\mathbf{b}(\theta, \mathbf{k})=\left[\begin{array}{cc}
\mathbf{a}(\theta) & \mathbf{0} \\
\mathbf{0} & \mathbf{a}^{*}(\theta)
\end{array}\right] \mathbf{k}
$$

where $\mathbf{k}$ is a $(2 \times 1)$ vector. Vector $\mathbf{k}$ allows to form a single steering vector from $\mathbf{a}(\theta)$ and $\mathbf{a}^{*}(\theta)$, and then it allows to exploit simultaneously the information provided by both the cyclic autocorrelation matrix and the cyclic conjugate autocorrelation matrix.

According to the subspace processing principle and by using this extended steering vector, the DOA of the SOI is given by the minima of the following function:

$$
\bar{P}(\theta, \mathbf{k})=\left\|\mathbf{U}_{n}^{H} \mathbf{b}(\theta, \mathbf{k})\right\|^{2}=\mathbf{k}^{H} \mathbf{M} \mathbf{k},
$$

where $\mathbf{M}$ is a $(2 \times 2)$ matrix:

$$
\mathbf{M}=\left[\begin{array}{cc}
\mathbf{a}^{H}(\theta) \mathbf{U}_{n 1} \mathbf{U}_{n 1}^{H} \mathbf{a}(\theta) & \mathbf{a}^{H}(\theta) \mathbf{U}_{n 1} \mathbf{U}_{n 2}^{H} \mathbf{a}^{*}(\theta) \\
\mathbf{a}^{T}(\theta) \mathbf{U}_{n 2} \mathbf{U}_{n 1}^{H} \mathbf{a}(\theta) & \mathbf{a}^{H}(\theta) \mathbf{U}_{n 2}^{*} \mathbf{U}_{n 2}^{T} \mathbf{a}(\theta)
\end{array}\right],
$$

and $\mathbf{U}_{n 1}$ and $\mathbf{U}_{n 2}$ are two submatrices of the same dimension

$$
\mathbf{U}_{n}=\left[\begin{array}{l}
\mathbf{U}_{n 1} \\
\mathbf{U}_{n 2}
\end{array}\right]
$$

It can be shown [3] that $\mathbf{U}_{n 2}^{*} \mathbf{U}_{n 2}^{T}=\mathbf{U}_{n 1} \mathbf{U}_{n 1}^{H}$. So, the two diagonal elements of the matrix $\mathbf{M}$ are equal. Note also that the two nondiagonal elements form a complex conjugate pair.

The minimum of the quadratic form in (9) for a particu$\operatorname{lar} \theta$ is given by the smallest eigenvalue of the matrix $\mathbf{M}$. This eigenvalue is always nonnegative since the quadratic form is nonnegative. When $\theta$ takes exactly the value of one of the true DOA, function (9) equals zero. Then, in this case, the smallest eigenvalue of $\mathbf{M}$ is equal to zero and the determinant of the matrix $\mathbf{M}$ equals zero too.

We now define the complex variable $z$ :

$$
z=e^{j(2 \pi \delta / \lambda) \sin (\theta)},
$$


where $\delta$ denotes the distance between two adjacent antennas and $\lambda$ denotes the wavelength of impinging SOI. Then $\mathbf{a}(\theta)$ can be written as

$$
\mathbf{a}(z)=\left[1, z, z^{2}, \ldots, z^{L-1}\right]^{T},
$$

and the matrix $\mathbf{M}$ is a function of $z$. We estimate the DOAs by finding the values of $z$ such that

$$
\operatorname{det}\{\mathbf{M}\}=0
$$

The left-hand side of (14) is a polynomial of $z$. The DOA estimation problem is then transformed into a polynomial rooting problem that can be solved using computationally efficient root-solving algorithms.

The polynomial of $z$ is given by the following form:

$$
\operatorname{det}\{\mathbf{M}\}=m_{11}^{2}-m_{12} m_{21}
$$

where

$$
\begin{aligned}
m_{11} & =\mathbf{a}^{T}\left(\frac{1}{z}\right) \mathbf{U}_{n 1} \mathbf{U}_{n 1}^{H} \mathbf{a}(z), \\
m_{12} & =\mathbf{a}^{T}\left(\frac{1}{z}\right) \mathbf{U}_{n 1} \mathbf{U}_{n 2}^{H} \mathbf{a}\left(\frac{1}{z}\right), \\
m_{21} & =\mathbf{a}^{T}(z) \mathbf{U}_{n 2} \mathbf{U}_{n 1}^{H} \mathbf{a}(z) .
\end{aligned}
$$

Therefore, $m_{11}$ is a polynomial in $z$ whose $l$ th coefficient is given by the sum of the elements of the $l$ th diagonal of $\mathbf{U}_{n 1} \mathbf{U}_{n 1}^{H}$, where $l=-L+1$ indicates the lowest diagonal and $l=L-1$ indicates the highest diagonal. Let $\mathbf{c}=\left[c_{1}, \ldots, c_{2 L-1}\right]^{T}$ be the column vector of the coefficients of the polynomial $m_{11}$. Then we have

$$
m_{11}=\left[z^{-L+1}, \ldots, z^{-1}, 1, z, \ldots, z^{L-1}\right] \mathbf{c}=\sum_{p=1}^{2 L-1} c_{p} z^{p-L}
$$

with

$$
c_{p}=\sum_{i=\max [1, L-p+1]}^{\min [L, 2 L-p]}\left[\mathbf{U}_{n 1} \mathbf{U}_{n 1}^{H}\right]_{i, p+i-L}
$$

Then, we can write that

$$
m_{11}^{2}=\left[z^{-L+1}, \ldots, z^{L-1}\right] \mathbf{c c}^{T}\left[z^{-L+1}, \ldots, z^{L-1}\right]^{T}
$$

Hence, the coefficients of the polynomial $m_{11}^{2}$ are equal to the sum of the antidiagonal elements of the matrix $\mathbf{c c}^{T}$. Let $\mathbf{s}=\left[s_{1}, \ldots, s_{4 L-3}\right]^{T}$ be the vector containing these $4 L-3$ coefficients. For $p=1, \ldots, 4 L-3$, we have

$$
s_{p}=\sum_{i=\max [1, p-2 L+2]}^{\min [2 L-1, p]}\left[\mathrm{cc}^{T}\right]_{i, p-i+1} .
$$

We obtain the following:

$$
m_{11}^{2}=\sum_{p=1}^{4 L-3} s_{p} z^{p-(2 L-1)}
$$

Since the matrix $\mathbf{U}_{n 1} \mathbf{U}_{n 1}^{H}$ is a Hermitian matrix, the elements of the vector $\mathbf{c}$ have the symmetry property $c_{i}=c_{2 L-i}^{*}$. Since $\operatorname{cc}^{T}$ is a symmetrical matrix, the coefficients of the polynomial $m_{11}^{2}$ keep the same property of symmetry $s_{p}=$ $s_{4 L-2-p}^{*}$.

In the same way, let $\mathbf{u}$ be the column vector containing the sum of the $2 L-1$ antidiagonal elements of the matrix $\mathbf{U}_{n 1} \mathbf{U}_{n 2}^{H}$ such that for $p=1, \ldots, 2 L-1$,

$$
u_{p}=\sum_{i=\max [1, p-L+1]}^{\min [L, p]}\left[\mathbf{U}_{n 1} \mathbf{U}_{n 2}^{H}\right]_{i, p-i+1} .
$$

Then, we can show that

$$
\begin{gathered}
m_{12}=\left[1, z^{-1}, \ldots, z^{-(2 L-2)}\right] \mathbf{u}, \\
m_{21}=\left[1, z, \ldots, z^{2 L-2}\right] \mathbf{u}^{*}, \\
m_{12} m_{21}=\left[1, \ldots, z^{-2 L+2}\right] \mathbf{u} \mathbf{u}^{H}\left[1, \ldots, z^{2 L-2}\right]^{T} .
\end{gathered}
$$

Let $\mathbf{r}$ be the column vector whose elements are the sum of the diagonal elements of the matrix $\mathbf{u u}^{H}$. For $p=1, \ldots, 4 L-$ 3 , the coefficients are

$$
r_{p}=\sum_{i=\max [1,2 L-p]}^{\min [2 L-1,4 L-p-2]}\left[\mathbf{u u}^{H}\right]_{i, p+i-(2 L-1)} .
$$

Hence,

$$
m_{12} m_{21}=\sum_{p=1}^{4 L-3} r_{p} z^{p-(2 L-1)}
$$

Since the matrix $\mathbf{u} \mathbf{u}^{H}$ is a Hermitian matrix, the coefficients of the polynomial $m_{12} m_{21}$ also have the property of symmetry $r_{p}=r_{4 L-2-p}^{*}$.

Equation (14) can now be written as

$$
\operatorname{det}\{\mathbf{M}\}=\sum_{p=1}^{4 L-3}\left(s_{p}-r_{p}\right) z^{p-(2 L-1)}=0
$$

The roots of the polynomial $\operatorname{det}\{\mathbf{M}\}$ can be computed using any polynomial rooting algorithm. The DOA estimates are obtained using (12):

$$
\theta_{k}=\arcsin \left(\frac{\lambda}{2 \pi \delta} \arg \left(z_{n}\right)\right)
$$




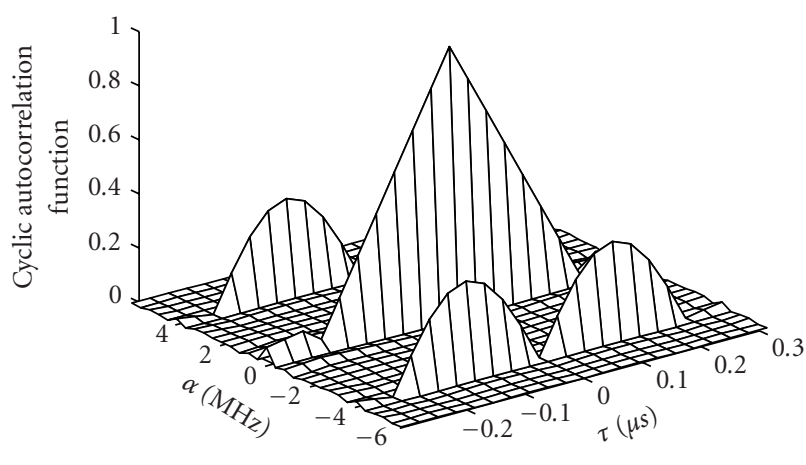

FIGURE 1: Magnitude of the BPSK cyclic autocorrelation function versus $\alpha$ and $\tau$.

where $z_{n}$ represents one of the $K_{\alpha}$ roots selected for DOA estimation. Due to the symmetry property of the polynomial coefficients, roots appear in reciprocal conjugate pairs $z_{i}$ and $1 / z_{i}^{*}$. In each pair, one root is inside the unit circle while the other is outside the unit circle (the two roots coincide if they are on the unit circle). Either one of the two can be used for DOA estimation, since they have the same angle in the complex plane. We have decided to use the roots inside the unit circle. According to (12), the modulus of the roots corresponding to incoming SOI should be equal to one. In the practice in presence of noise, modulus are not necessary one but are expected to be close to one. We then select the $K_{\alpha}$ roots that are the nearest to the unit circle as being the roots corresponding to the DOA estimates.

Note that the degree of the polynomial $\operatorname{det}\{\mathbf{M}\}$ is $4 L-4$ ( $4 L-3$ coefficients). Hence, the number of roots is $4 L-4$, and since the roots appear in reciprocal pairs, the proposed procedure allows in some conditions (e.g., BPSK, AM signals), to determine until $2(L-1)$ signals. This point should be emphasized since the number of signals estimated by the proposed method may be larger than the number of sensors. This characteristic is due to our proposed data model.

\section{SIMULATION RESULTS}

In this section, we present some simulation results that illustrate the performance of the proposed algorithm. We also compare the simulation results of the proposed procedure with those of the classical root-MUSIC algorithm.

We consider here a linear uniformly spaced array with 6 sensors spaced by a half wavelength of the incoming signals. Incoming BPSK cyclostationary signals are generated with noise, and the signal-to-noise ratio (SNR) is $0 \mathrm{~dB}$ for each signal. The bit rate of the BPSK SOI is $4 \mathrm{Mbps}$. Other signals are considered as interferers, which are BPSK modulated signals with a $3.2 \mathrm{Mbps}$ bit rate. In order to choose correctly the parameters $\alpha$ and $\tau$, we have estimated the magnitude of the cyclic correlation function (Figure 1) for a 4 Mbps BPSK modulated signals sampled with the frequency $32 \mathrm{MHz}$ during 25 microseconds. It can be noted that the magnitude of the cyclic autocorrelation function and that of the conjugate cyclic autocorrelation function are

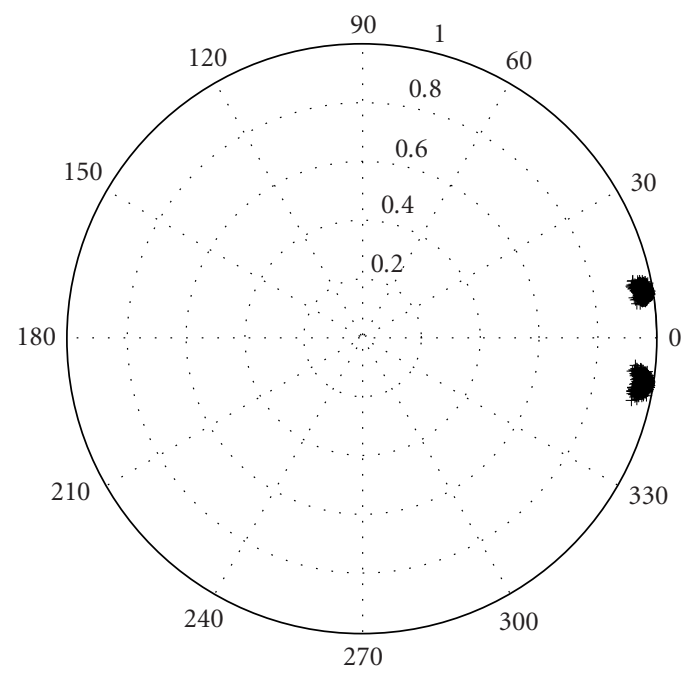

FIGURE 2: Selected polynomial roots by the proposed method.

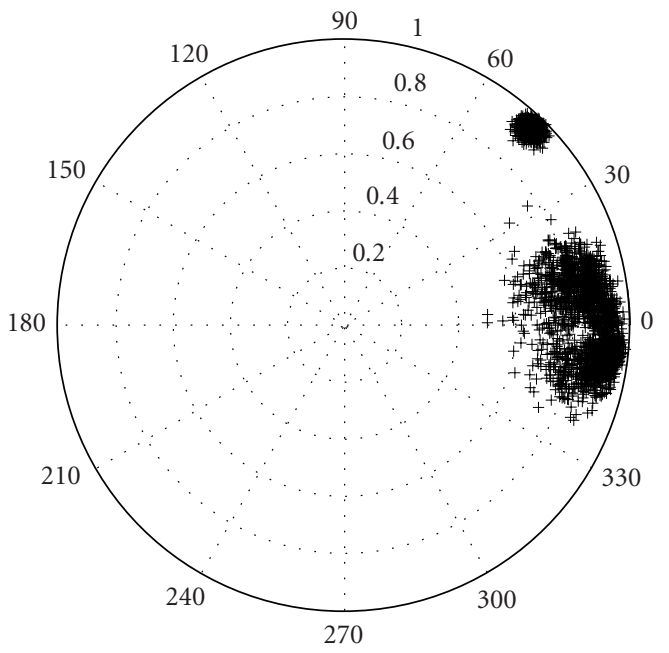

FIGURE 3: Selected polynomial roots by the classical root-MUSIC method.

equal for a BPSK signal. According to this result, the proposed cyclic method is simulated with $\alpha=4 \mathrm{MHz}$ and $\tau=$ 0.125 microseconds. In the next simulations, the averaging time is equal to 25 microseconds and the sample frequency is $32 \mathrm{MHz}$. The contribution of both the interferer signals and that of the noise are theoretically zero in the two cyclic correlation matrices.

The behavior of the proposed method and that of the classical root-MUSIC method can be compared by drawing the selected roots by these two methods in the polar plane. Figure 2 shows the distribution of the selected roots by the proposed method obtained from 500 Monte Carlo simulations when two BPSK SOI arrive from $-3^{\circ}$ and $3^{\circ}$, and one interferer BPSK source from $15^{\circ}$ DOA. Figure 3 shows the distribution of the selected roots by the classical root-MUSIC method obtained from 500 Monte Carlo simulations in the same situation. 
TABLE 1: Mean and standard deviations for both methods.

\begin{tabular}{lcccc}
\hline \multirow{2}{*}{ Method } & True DOAs $\left(^{\circ}\right)$ & SOI \#1 & SOI \#2 & Interferer \\
& & $-3^{\circ}$ & $3^{\circ}$ & $15^{\circ}$ \\
\hline \multirow{2}{*}{ Proposed method } & Mean & -2.973 & 3.009 & - \\
& Standard deviation & -0.355 & 0.260 & - \\
\hline \multirow{2}{*}{ Root method } & Mean & -2.583 & 2.635 & 14.907 \\
& Standard deviation & 1.151 & 1.828 & 0.429 \\
\hline
\end{tabular}

TABle 2: Mean and standard deviations for the proposed method with seven SOI and seven interferer signals.

\begin{tabular}{c|ccc}
\hline SOI & True DOAs $\left(^{\circ}\right)$ & Mean & Std. deviation \\
\hline SOI \#1 & -70 & -69.649 & 1.012 \\
SOI \#2 & -50 & -49.986 & 0.512 \\
SOI \#3 & -30 & -30.005 & 0.442 \\
SOI \#4 & -10 & -10.005 & 0.289 \\
SOI \#5 & 20 & 19.989 & 0.623 \\
SOI \#6 & 40 & 40.017 & 0.542 \\
SOI \#7 & 60 & 59.945 & 0.734 \\
\hline
\end{tabular}

These figures show that the proposed cyclic method allows to perfectly select the two SOI, and ignores the interferer signal. The polynomial roots selected by the proposed cyclic method are all located in two small areas. Those obtained from the classical root-MUSIC are more scattered. It can be deduced that the estimation provided by the proposed method is more accurate.

The performance of the estimators can also be obtained from 500 Monte Carlo simulations by calculating the mean and the standard deviation of DOA estimates. Table 1 shows the simulation results when two BPSK SOI arrive from $-3^{\circ}$ and $3^{\circ}$, and one interferer BPSK source from $15^{\circ}$ DOA.

The proposed cyclic algorithm performs better than the classical root-MUSIC thanks to the exploitation of both cyclic correlation matrices; more information about sources is used and the observation dimension space (i.e., the size of the extended covariance matrix $\left.\mathbf{R}_{C E}^{\alpha}(\tau)\right)$ is doubled.

Table 2 provides simulation results for only the proposed procedure when seven SOI and seven interferer BPSK sources impinge on the 6-sensors array. DOAs of SOI are $-70^{\circ},-50^{\circ}$, $-30^{\circ},-10^{\circ}, 20^{\circ}, 40^{\circ}$, and $60^{\circ}$. DOAs of interferer signals are $-35^{\circ},-20^{\circ}, 0^{\circ}, 15^{\circ}, 25^{\circ}, 30^{\circ}$, and $45^{\circ}$. The method always ignores interferer signals and gives rather accurate estimations of the SOI DOA. These last results show that the proposed method is signal selective and is able to handle more sources of interest than the number of sensors.

\section{CONCLUSION}

We have described a signal-selective procedure for DOA estimation. By assuming that the incoming signals are BPSK modulated signals, the algorithm uses the cyclostationary property of the signals to improve the estimations performance. The proposed method is able to handle more sources of interest than the number of sensors. Moreover, by using a polynomial rooting technique, the proposed algorithm does not require an explicit search procedure, and hence considerably reduces the computational requirements.

\section{REFERENCES}

[1] W. A. Gardner, Cyclostationarity in Communications and Signal Processing, IEEE Press, New York, NY, USA, 1993.

[2] W. A. Gardner, "Simplification of MUSIC and ESPRIT by exploitation of cyclostationarity," Proceedings of the IEEE, vol. 76, no. 7, pp. 845-847, 1988.

[3] P. Chargé, Y. Wang, and J. Saillard, "An extended cyclic MUSIC algorithm," IEEE Trans. Signal Processing, vol. 51, no. 7, pp. 1695-1701, 2003.

[4] P. Chargé, Y. Wang, and J. Saillard, "A non-circular sources direction finding method using polynomial rooting," Signal Processing, vol. 81, no. 8, pp. 1765-1770, 2001.

[5] A. J. Barabell, "Improving the resolution performance of eigenstructure-based direction-finding algorithms," in Proc. IEEE Int. Conf. Acoustics, Speech, Signal Processing (ICASSP '83), vol. 8, pp. 336-339, Cambridge, Mass, USA, April 1983.

[6] M. Wax and T. Kailath, "Detection of signals by information theoretic criteria," IEEE Trans. Acoustics, Speech, and Signal Processing, vol. 33, no. 2, pp. 387-392, 1985.

Pascal Chargé was born on January 2, 1973. $\mathrm{He}$ received the $\mathrm{Ph} . \mathrm{D}$. degree in electrical engineering from the University of Nantes, France, in 2001. Until 2003, he was with the IRCCyN Laboratory, where he was a Research Fellow with the Electronic Systems, Telecommunications, and Signal Processing Division. Since September 2003, he has been an Assistant Professor at the National Institute of Applied Sciences (INSA),

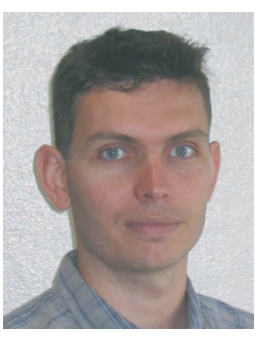
Toulouse, and he is Research Fellow with the LESIA Laboratory. His research interests include sensors array processing, statistical signal processing, and signal processing for wireless communications.

Yide Wang received the B.S. degree in electrical engineering from Beijing University of Post and Telecommunication (BUPT), Beijing, China, in 1984, the M.S. and the Ph.D. degrees in signal processing from the University of Rennes, France, in 1986 and 1989, respectively. He is now a Professor at the Polytechnic School, the University of Nantes, France. His research interests include array processing, spectral analysis, and mobile wireless communications systems.

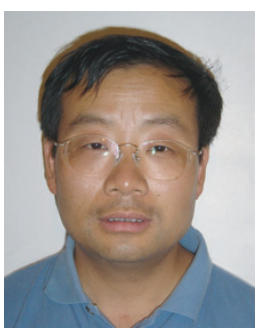

